\title{
Science with coffee and hobnobs
}

\section{Hannah Foreman, Averil Macdonald, Robert Eason}

Hannah D. Foreman, Averil M. Macdonald, Robert W. Eason, "Science with coffee and hobnobs," Proc. SPIE 9664, Ninth International Topical Meeting on Education and Training in Optics and Photonics, 96641S (24 October 2005); doi: $10.1117 / 12.2207704$

SPIE Event: Ninth International Topical Meeting on Education and Training in Optics and Photonics, 2005, Marseille, France 


\title{
Ref ETOP043
}

\section{Science with coffee and Hobnobs}

\author{
Hannah D. Foreman ${ }^{1 *}$, Averil M. Macdonald ${ }^{2}$, Robert W. Eason ${ }^{3}$.
}

${ }^{1}$ School of Physics and Astronomy, University of Southampton, Southampton, UK.

${ }^{2}$ Department of Physics, University of Reading, UK.

${ }^{3}$ Optoelectronics Research Centre, University of Southampton, Southampton, UK.

\begin{abstract}
Many parents or guardians of primary school pupils have little knowledge of science, and many lack confidence in their ability to help their children, though most welcome the chance to do so. We describe our experiences running a series of meetings in the form of coffee sessions at local primary schools, where parents can increase their knowledge and confidence in the science their children study, and engage in simple experiments with their children to apply the knowledge they gain. We discuss how this programme can be instrumental in improving the profile of scientific education and scientific careers for children of a young age.
\end{abstract}

\section{Keywords}

National curriculum science, Primary school children, Parents/guardians, optics outreach.

\section{Introduction}

\section{Summary}

The following paper describes the use of coffee morning/afternoon sessions to communicate the optics part of the UK National Curriculum [1] to parents and guardians of primary school children. It is internationally recognised that both school and parents have crucial roles to play in a child's learning and the impact is greater if they work in partnership [2]. We will outline the benefits and the problems of running these interactive sessions within the primary school teaching curriculum. Some of these challenges include communicating effectively with a group of adults with a wide range of educational backgrounds, supplying a range of practical activities and experiments that cater to the needs of children within the wide primary age span within one session, managing time and equipment limitations, and the challenge of producing a concise handout to accompany the series. The benefits of this scheme, which we believe far outweigh the problems, include an improved knowledge and understanding of basic optical concepts for both parents and children, an increase in the confidence of parents to communicate scientific ideas and discuss scientific concepts with their children, improvements in parent and student motivation, and the opportunity for parents and pupils to meet active researchers from the University community to hear about the latest research and to ask them questions.

\section{Background and Programme}

This project follows the success of a small pilot programme entitled 'Particle Physics with Coffee and Hobnobs' funded through a Particle Physics and Astronomy Research Council (PPARC) Public Awareness Grant in early 2003. This pilot demonstrated an appetite amongst parents of primary school pupils for information and knowledge to support them as they seek to help their children with their science work at Key Stages 1 and 2 (ages 5-7 and 7-11) of the UK National Curriculum. The 'Science with Coffee and Hobnobs' optics sessions we describe were run in the 
primary school of the child, publicised to the parents by the child's teacher. The sessions are funded by a Partnership for Public Awareness of Science award (PPA) from the Engineering and Physical Sciences Research Council (EPSRC). They are led by a professional educational consultant (AMM), an academic member of staff from the University of Southampton (RWE), and several postgraduate student volunteers researching in the areas of optoelectronics, and laser physics at the University of Southampton (HDF). The meetings attract between 20 and 40 parents, and their corresponding children. All the material is condensed into one session, lasting 1.5 hours, with half of this time devoted to adult briefing, and the other half to parent-child experimentation.

During the sessions, an overview of the UK National Curriculum is given to parents by the educational consultant, explaining both the relevant content, and the mental blocks that children sometimes have when learning about the topics in question. Parents are supplied with a handout summarising the material covered, explaining the science, and suggesting some activities to pursue at home to help their children grasp the concepts discussed throughout the session (figure 1a). The university academic then explains how these basic ideas relate to research carried out at the University, and technology present in everyday life. The children then join their parents for an interactive experimentation session, helped by the consultant, the academic and the postgraduate volunteers, to initiate and inspire further joint investigations and involvement at home.

\section{Topics and Activities Covered}

The optics topic within the Science UK National Curriculum is covered at Key Stage 1 (KS1) in Year 1 (age 5-6) where pupils are introduced to the concepts of light and dark, and again at Key Stage 2 (KS2) in Year 5 (age 9-10), where light and shadows are addressed. During the sessions, parents are familiarised with vocabulary that they may find useful to communicate some of the basic principles with their children. Words such as light, bright, dark, black, night, day and reflect are highlighted for KS1, and transparent, opaque, shadow and blocking light for KS2. The basic ideas that their children should be grasping are explained to the parents. At $\mathrm{KS} 1$, these include that light comes from a source, it is impossible to see in the dark, and that in order for us to be able to see an object, it either emits, or reflects light. At KS2, children begin to recognise how shadows are formed and that shadow length changes over the day as the position of the sun changes.

Some common misconceptions that children may have are explained to the parents to give them some feel for how their child is thinking. For example, children often think 'light is just there' and don't think about where it comes from. The fact that you can't see light travel $f$ rom one place to another because of its speed reinforces this notion. Moreover, many children confuse their shadows with their reflection in a mirror, and will often draw a shadow with a face on. The idea of shadows forming helps the child accept the idea that light travels away from a light source [3]. We suggest activities for parents to carry out with their children to overcome and reason through these misconceptions. 

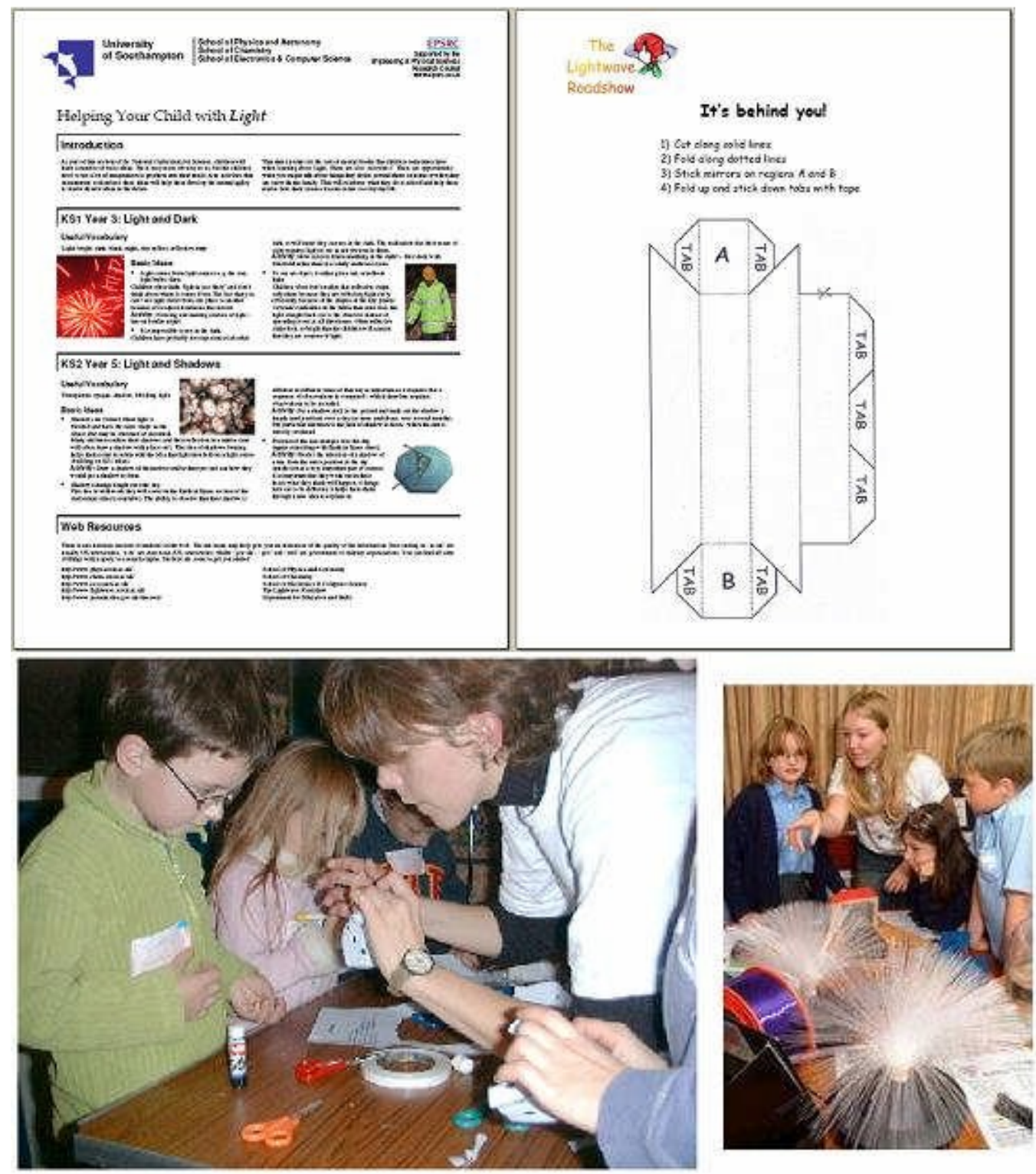

Figure 2: Photographs from various sessions showing a) periscope making and b) optical fibre lamp demonstrations. 
Opportunities when parents might discuss things that children notice around them, at home, or on family outings aresuggested, such as encouraging children to notice and name sources of light, and predict where their shadow will fall, as well as more in-depth activities such as making a sundial in the garden. Parents are encouraged to reinforce what the children learn at school, and help them to realise how their science lessons relate to everyday life using this range of ideas, and extending into their own initiatives.

The set of demonstrations and experiments used during the 'light' sessions are derived from those used in the University of Southampton's 'Lightwave Roadshow' (www.lightwave.soton.ac.uk), a photonics outreach project run by volunteers from the Optical Society of America Student Chapter based at the University [4]. Students and parents are encouraged to make a periscope together with materials provided by the Lightwave Roadshow and use it to view each other across a table, engaging an element of fun into the activity for even the youngest children who delight in seeing the image of a loved one in something they helped to make (figure $1 \mathrm{~b}$ and $2 \mathrm{a}$ ). The act of making the periscope, then having the opportunity to use it encourages children and parents to think about the key scientific principles behind its operation. The postgraduate helpers encourage discussion about reflection, and hint at the use of ray diagrams to understand the path that light takes from an object to our eye.

A shadow-painting rig is set up using a simple household desk-lamp. Parents and pupils are encouraged to draw around each other's shadows, demonstrating that no elaborate equipment is necessary, and encouraging interaction between child and parent in a scientific activity. This often attracts a small audience, ideal for initiating discussions about how shadows are formed. It is common for children to then embellish their shadow pictures with facial features, an excellent moment to point out the difference between a shadow and a reflection, and for parents to see the common misconceptions that have been highlighted in the earlier session. The children take their pictures away afterwards, providing a reminder of the concepts the task encourages them to think about, and also providing a medium for parents to engage further discussion and activities at home.

Other commercially available optics toys, and common household objects are used to demonstrate simple optical principles such as reflection and the spectrum providing a platform for communication of the basic concepts covered in Key Stages 1 and 2 without the use of imposing equipment. An emphasis is placed on parent-child interaction, and enjoying this experimentation, demonstrating the accessibility of these simple experiments and demonstrations in the home. Colour filters can be made from transparent sweet wrappers, diffraction gratings from the under-side of CDs, and distorted mirrors from kitchen foil. Kaleidoscopes are amongst the demonstrations, and many parents confess that they have never considered the optics behind this familiar childhood toy. Optical fibre lamps, often used for home decoration, and also some Christmas trees are also amongst the kit, and the guided light principles behind their operation are explained and related to the optoelectronics research at the university (figure $2 b$ ).

\section{The Challenges}

The main challenges for this scheme arise from the issues of communicating effectively with the mixed audiences of adults that the sessions attract. Their own misconceptions and resistances must be overcome initially, without allowing the sessions to be perceived as either inaccessible or patronising. In order to do this, an informal atmosphere is generated by serving coffee and biscuits as the parents arrive and arranging the chairs in a circle rather than in a formal lecture 
style. The initial address given by the educational consultant is presented from the point of view of a parent and the language used is colloquial, while elaborate scientific terms are avoided, for example, we talk about 'light' instead of 'optics'. Likewise, the handouts provided are written in a relaxed style, using simple colloquialisms, and avoiding scientific language. Plenty of everyday examples and scenarios are talked about, drawing on the team's own experiences, and relaxed questioning is encouraged.

The logistical problem of supplying a range of practical activities and experiments that cater to the needs of children within the wide primary age span in one session is overcome by the use of postgraduate volunteers. They explain the equipment to individual parent-pupil pairs, tailoring their explanation to each different pair's level. The volunteers gauge how far to take the discussion on an individual scale, sometimes merely communicating the basics, and other times, engaging in detailed discussions about their own research, and answering varied questions from both children and parents. Emphasis is placed on the fun element of the activities for all, and involvement of parent and child together is strongly encouraged (figure 3 ).

Naturally, the time dedicated to each session is limited by the length of the school day, and the amount of time that parents have to spare, as well as the amount of time over which it is reasonable to remove children from the classroom. To overcome this, the session is timed to coincide with the beginning of the school day, or the end, so that parents can attend just after or before taking their child to or from school. The children are only present for the experimental part of the session, avoiding any issues with them getting bored within the 'adult' parts of the session, and keeping to a minimum the time they are absent from lessons. No teacher presence at the session is required so there is no strain on teaching resources, though experience has shown that teachers often prefer to attend as they report that they learn a great deal from the sessions. The problem of inaccessibility of optics equipment is overcome by using household items to show how accessible home-experimentation can be.

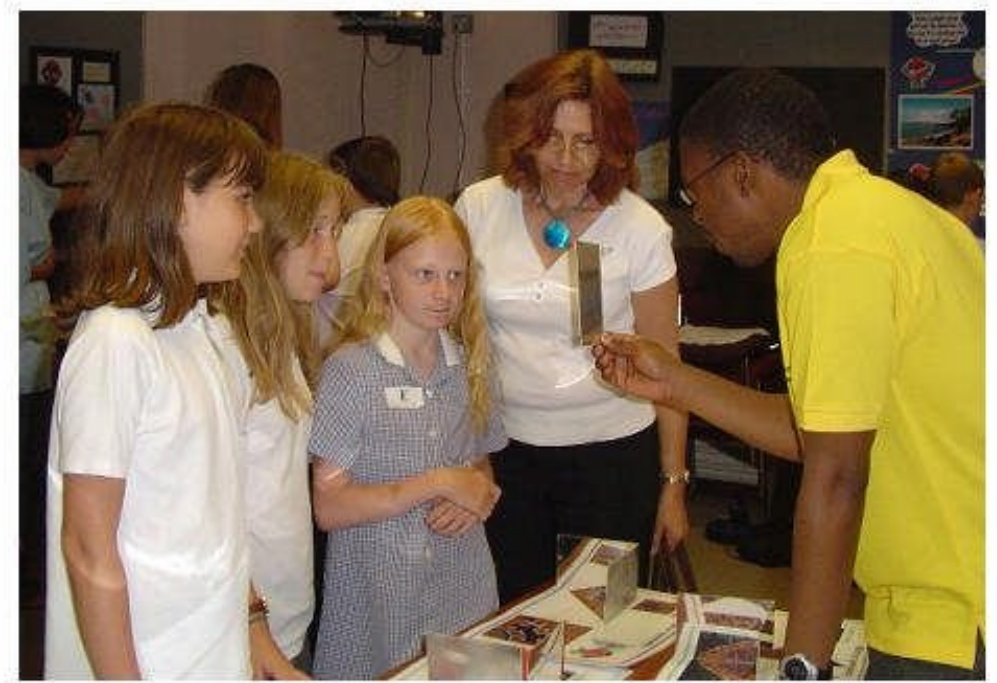

Figure 3: Parent, children, and postgraduate volunteer carrying out some reflection experiments with mirrore 
A further limitation of these sessions is our ability to gauge how successful they are. Teachers are very forthcoming with positive feedback, and parents often make comments at the end of the session. Moreover, questionnaires are available, and parents are encouraged to complete one, however, it must be recognized that some of the parents in attendance may have limited literacy skills, if any at all, or may mistake the questionnaire as more of a test. Consequently the questionnaires are kept brief, and cannot be imposed upon the audience. Furthermore, although we anticipate that the sessions will have a positive effect on children's' perception of science later into their education and careers, it is not within the scope of this project to carry out research in the future to see if we have positively influenced these particular children.

\section{The Benefits}

Parents of primary age pupils are an extremely influential group of people. Their attitude to science can make a big difference to the way that their children in turn perceive science, and their likelihood of pursuing it for future study and careers [5]. By inviting parents to an informal meeting held in the familiar school environment, they are more likely to feel comfortable than in a formal lecture theatre. Evidence shows that parents are naturally keen to help their children [6]. This is their motivation to attend these meetings. However, once the ice is broken and they are confident with the basic science, they are found to be very open to hearing more about science beyond the UK National Curriculum. This project offers a very effective means of entering into dialogue with an audience who may not normally attend science-based meetings, not only promoting the immediate scientific education of their children, but also highlighting the importance of science through secondary education, and raising awareness of the option to continue into higher education, and even careers.

Family learning has been found to benefit both child and adult in improved literacy and numeracy for each, increased confidence in the education system for parents, and increased acceptance and pleasure in learning for the child [7]. These sessions provide a basis to communicate optics to a generation who may have received no formal scientific education. Often, a barrier to parent-child interactions in science education is a parent's lack of confidence in their own knowledge, or a resistance of the parent to being open to learning because of previous bad experiences during their own schooling. These sessions overcome these problems in their presentation as a project to help parents help their children; it may in fact be an education for them also. 


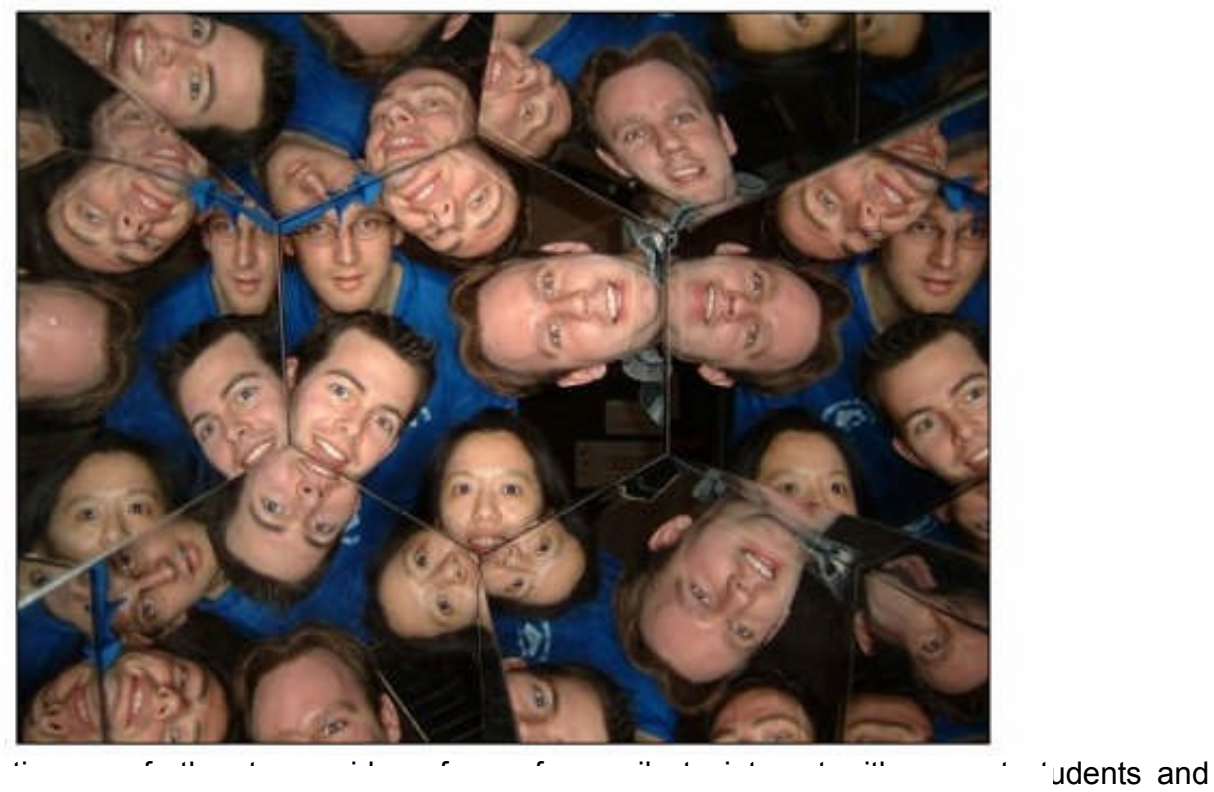

The

Figure 4: role model postgraduate volunteers playing with the Lightwave kit. that by the age or 11 , most cnılaren nave tormea the opınıons ana aspıratıons that WIII determine their likelihood of pursuing education beyond the age of 16 [8]. It is hoped that the insight into higher education provided by these sessions will provide a positive influence. The postgraduate student volunteers act as positive role models for the pupils, challenging the archetypal image that many children and adults attribute to scientists (figure 4). Moreover, a conscious effort is made to include female postgraduate volunteers to raise the profile of women within science. This is also of benefit to the volunteers as they are given the opportunity to develop their ability to communicate science at different levels, and reaffirm their basic skills and knowledge in what have proven to be very rewarding sessions. The programme is also of benefit to the University in its development of communication with the surrounding community, working to raise the University's profile, and awareness of its extensive research activities as well as its educational programmes.

\section{Conclusion}

We have demonstrated a simple scheme to overcome the problems of encouraging parental involvement in primary optics education. Using accessible meetings as a guide to the UK National Curriculum, we not only enrich children's scientific education through parental involvement and joint activities, but also aim to affirm and improve scientific knowledge of the attending parents. Furthermore, the sessions provide a forum for interaction between active photonics researchers and parents to improve the profile of scientific research and careers within this group with a view to improving the profile of science within the whole family. 


\section{References}

1. Qualifications and Curriculum Authority, The National Curriculum Online, www.nc.uk.net

2. The Scottish office, Parental Participation in schools, (2003)

3. DfES, The Standards Site, www.standards.dfes.gov.uk

4. D. Guilhot, J. Baggett and I. Musgrave, University of Southampton Takes Science Into the Schools, Optics \& Photonics News, 13 \#8, pp 16-17, (2002)

5. K. J. Topping, C. Peter, P. Stephen and M. Whale, Cross-Age Peer Tutoring of Science in the Primary School: Influence on scientific language and thinking, Educational Psychology, 24 \#1, (2004)

6. B. Williams, J. Williams and A. Ullman, Parental involvement in education, DfES research report 332 (2002)

7. Ofsted, Family learning: a survey of good practice, (2000)

8. P. Croll, The Formation and Transmission of Educational Values and Orientations, ESRC award R000239963 (2005)

* hdf@phys.soton.ac.uk, tel: +44 (0)23 8059 2066, fax: +44 (0)23 8059 3910, http://www.hep.phys.soton.ac.uk/hycs/ 